\title{
Lipoma Arborescens of Knee: A Case Report
}

\author{
Ruchit Khera*,Hardas Singh Sandhu**,Veushj Sharma***, \\ Parvinder Singh Sandhu*** \\ Dr.Hardas Singh Orthopaedic Hospital And Super Speciality Research Centre, Circular Road, \\ Amritsar, Punjab, INDIA \\ *Presenting Author / Post-Graduate Resident, Department Of Orthopaedics. \\ **Head Of Department, Department Of Orthopaedics. \\ ***Consultant Orthopaedic Surgeon.
}

\begin{abstract}
Lipoma Arborescens is a lesion arising from synovium and is characterized by diffuse increase in the quantity of subsynovial fat that bulges the overlying synovial lining and produces a villous architecture. It is a rare tumour. There is a fatty villonodular proliferation of the synovium predominantly in the knee, although its origin from other joints has also been reported. Though of unknown etiology, it is usually seen secondary to articular / peri-articular trauma, or synovitis. We report a case of 56 years old male who presented with unilateral monoarticular swelling associated with chondromalacia and subchondral cyst of the left knee. A large tumor measuring $17 x 15 \mathrm{~cm}$ extending from suprapatellar area to anterior lower thigh was excised. The largest Lipoma Arborescens tumor ever reported was of the size $14 x 11 \mathrm{cms}$ and as per our research, lipoma Arborescens reported in our study is the largest ever reported. Its etiopathogenesis, histopathological and radiological findings are discussed alongwith.
\end{abstract}

Keywords: Lipoma Arborescens, Synovial Tumor, Fatty villonodular proliferation

\section{Introduction}

Lipoma Arborescens is a rare benign lesion of intra-articular origin and is characterized by diffuse replacement of synovial tissue by mature adipocytes, causing a villous lipomatous proliferation of the sinovial membrane. ${ }^{1}$ The term 'Arborescens' originates from the Latin word 'arbor' meaning tree, thus describing the tumor's characteristic tree-like appearance. Histologically, it is characterized by villous, polypoid, and lipomatous proliferation of the synovium with the subsynovial layer replaced by fat cells. This tumor usually occurs in the knee but there are reports of involvement of other joints such as the hip, ankle, shoulder, elbow and wrist. Presentation is usually monoarticular however polyarticular involvement has also been reported including bilateral involvement ${ }^{3}$. The primary cause of lipoma arborescens remains unknown; however associated conditions have been reported which include local trauma, meniscal injuries, psoriatic arthritis, osteoarthritis, rheumatoid arthritis, diabetes mellitus and gout.In all, 113 papers were reviewed for Lipoma arborescens. All the abstracts were read by single author (RK) and relevant papers were reviewed. In a case report study by Prabhakar V, incidence of Lipoma Arborescens was reported to be between 0.14-0.25\%.

\section{Case Report}

A 56 year old male presented with 1 year history of gradually progressing swelling around knee and distal thigh; several episodes of exacerbation of pain. He had no history of catching, locking, or giving away of knee, There were no constitutional symptoms or history of any injury. The patient had been treated with physiotherapy and non steroidal anti-inflammatory drugs despite which the symptoms gradually increased in severity. (Fig.1: Pre operative clinical picture showing suprapatellar swelling extending into distal thigh)

Physical Examination revealed soft to firm boggy swelling over anterior lower third thigh involving suprapatellar pouch, with slippery edges, free from overlying skin and underlying tissues. Medial Joint line tenderness was present. Pivot Shift, McMurrays and Lachman Tests were negative. Plain radiograph of Knee with thigh (antero-posterior and Lateral views) revealed multiple lobulated calcified loose bodies along with radiolucent soft tissue shadow extending from suprapatellar pouch into anterior thigh. (Fig.2: Radiographs of Knee) MRI revealed a large soft tissue mass in suprapatellar area extending upto lower thigh with high signal intensity on T1 and T2 weighted images. (Fig.3: MRI Knee with Thigh) Open Surgical Excision of mass with synovectomy was done. Through anterior mid line para-patellar approach, a large fused mass of loose bodies filled with lipomatous tissue arising from knee with synovial extending to the lower third thigh, measuring $17 \times 15 \mathrm{~cm}$ was revealed. Chondromalacia and sub-chondral cysts were also visualised. (Fig.4: Per-operative pictures of Knee) (Fig.5(a) and 5(b): Excised Tumor) Excised tumor was subjected to histopathological examination which show papillary fronds with infiltration by mature adipocytes. Fronds were lined on the surface by synovium. The synoviocytes showed reactive changes, increased amount of eosinophillic cytoplasm. 
Sections in additions show small blood vessels proliferation with infiltration of parenchyma by mononuclear inflammatory cells seen as mainly lymphocytes and few plasma cells. Also noted were small foci of calcification. (Fig.6: Histopathological Slide)

\section{Discussion}

Tumors of the synovium are rare and can involve the lining of the joints, tendons, or bursa. Lipoma arborescens is a rare benign synovial tumor with villous lipomatous proliferation of the synovial membrane. It has been reported in patients of almost all age groups and is equally common in both the sexes. The exact etiopathogenesis of Lipoma arborescens is not known; majority of cases arise de novo. Two types of Lipoma Arborescens have been described in literature; Primary and Secondary. The primary type is rare and could be due to hereditary conditions. The secondary type is due to chronic irritation of synovium resulting from repeated trauma. Thus, chronic conditions like degenerative joint diseases, trauma, chronic rheumatoid arthritis, psoriasis, gout, diabetes mellitus or a popliteal cyst may trigger formation of Lipoma arborescens. Plain radiograph are non specific for Lipoma Arborescens but may show soft tissue density or calcified loose bodies or signs of underlying disease. Magnetic Resonance Imaging is diagnostic non-invasive modality and shows high signal intensity of fat on both T-1 and T-2 weighted images with frond like architecture.

Grossly, tumor has a frond-like appearance with multiple broad-based polypoid or thin papillary villi composed of fatty yellow tissue which may be calcified. Mircroscopically, the villi are filled with mature adipose cells, and enlarged or congested hyperaemic capillaries may be present. The overlying synovial membrane may contain mononuclear chronic inflammatory cells and the synovial cells may appear to be enlarged and reactive, with abundant eosinophilic cytoplasm. Importance lies in distinguishing it from Hoffa disease and synovial lipoma. Hoffa disease is a syndrome of infra-patellar fat pad impingement. It results from trauma to fat pad which incites acute inflammatory response resulting in impingement of involved fat pad between articular surfaces i.e patello-femoral or tibio-femoral joints resulting into enlarged fat pad with hemorrhagic areas and necrosis within it resulting in fibrosis with tumor like appearance. Hoffa disease shows low signal intensity on T1 and T2 weighted MRI sequences. ${ }^{8}$ Synovial lipoma is mainly seen in Knee Joint especially in suprapatellar pouch usually resulting from overgrowth of fat within intraarticular synovial tissue. Similar to Lipoma Arborescens, synovial lipoma also show high intensity signals on T1 and T2 weighted images on MRI, and almost identical microscopic features. ${ }^{9}$ Two can be distinguished by macroscopic appearance. Synovial lipoma is usually intraarticular, small in size, yellowish in colour, is a solitary polyp-like mass, round to oval in shape, with a short stalk; however lipoma arborescens appears as a large, frond-like mass. Importance in differentiating three diseases is that treatment of choice for Hoffa Disease and synovial lipoma is arthroscopic or open localised resection whereas treatment of choice for Lipoma Arborescens is arthroscopic synovectomy or open synovectomy with excision of tumor mass. Recurrence following synovectomy is has not been reported.

\section{Conclusion}

Lipoma arborescens is a benign synovial neoplasm which can arise denovo or secondary to other underlying causes. Although rare lipoma arborescens should be kept in mind as differential diagnosis in longstanding swelling of large joints.

\section{References}

[1]. Davies AP, Blewitt N. Lipoma arborescens of the knee. Knee 2005;12(5):394-6.

[2]. Murphey MD, Carroll JF, Flemming DJ, Pope TL, Gannon FH, Kransdorf MJ. From the archives of the AFIP: Benign musculoskeletal lipomatous lesions. Radiographics 2004;24(5):1433-66.

[3]. Yan CH, Wong JW, Yip DK. Bilateral knee lipoma arborescens: a case report. J Orthop Surg (Hong Kong) 008;16(1):107-10

[4]. Wolf R, Zoys G, Saldivar V, Williams R. Lipoma arborescens of the hip. Am J Orthop. 2002;31(5):276-9.

[5]. Chae EY, Chung HW, Shin MJ, Lee SH. Lipoma arborescens of the glenohumeral joint causing bone erosion: MRI features with gadolinium enhancement. Skeletal Radiol 2009;38(8):815-8.

[6]. Chaljub G, Johnson PR. In vivo MRI characteritics of lipoma arborescens utilizing fat suppression and contrast administration. J Comput Assist Tomogr 1996;20:85-7.

[7]. Prabhakar V, Naik S. Lipoma arborescens of the knee: Report of a case with a full range of motion and literature review. Saudi J Sports Med 2014;14:55-8.

[8]. Metheny JA, Mayor MB. Hoffa disease: Chronic impingement of the infrapatellar fat pad. Am J Knee Surg 1998;1:134-39.

[9]. Matsumoto K, Okabe H, Ishizawa M, Hiraoka S. Intraarticular lipoma of the knee joint. A case report. J Bone Joint Surg Am 2001;83-A (1):101-5. 


\section{Figure}

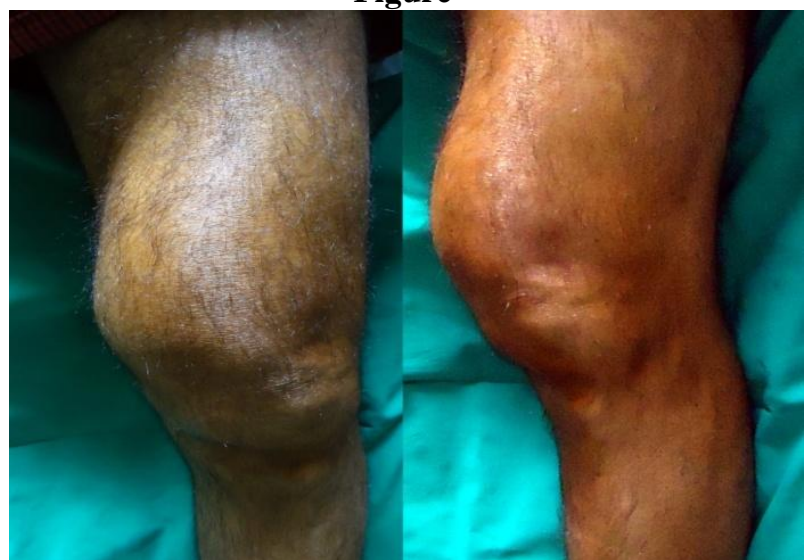

Fig -1

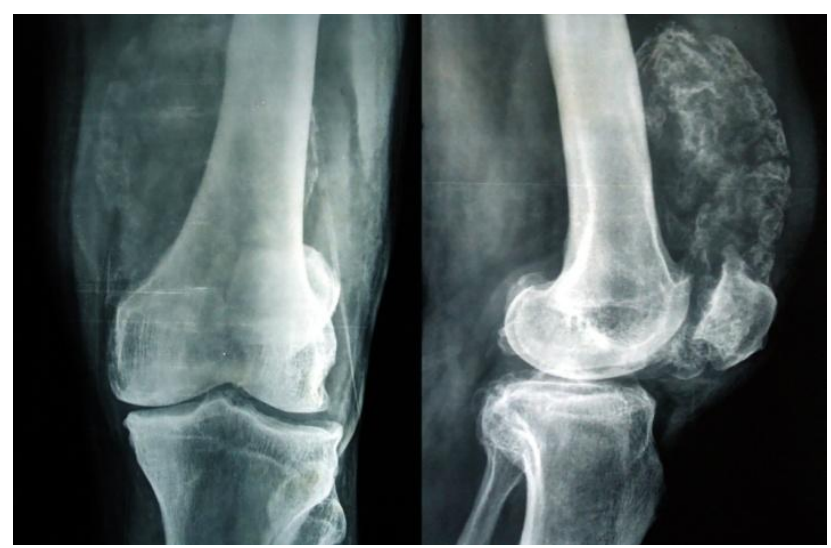

Fig -2
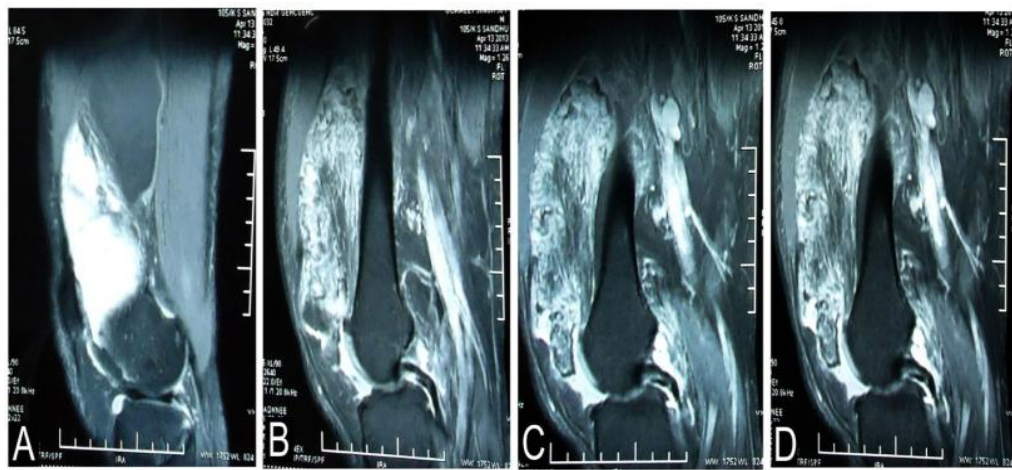

Fig -3

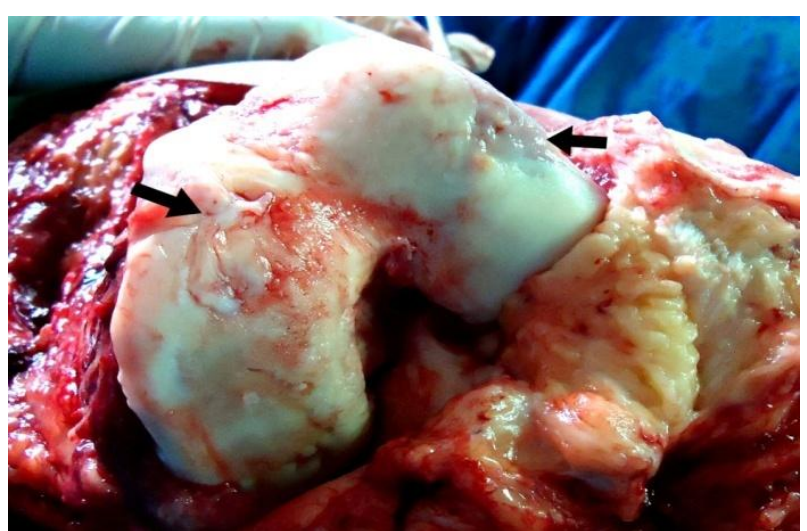

Fig - 4 


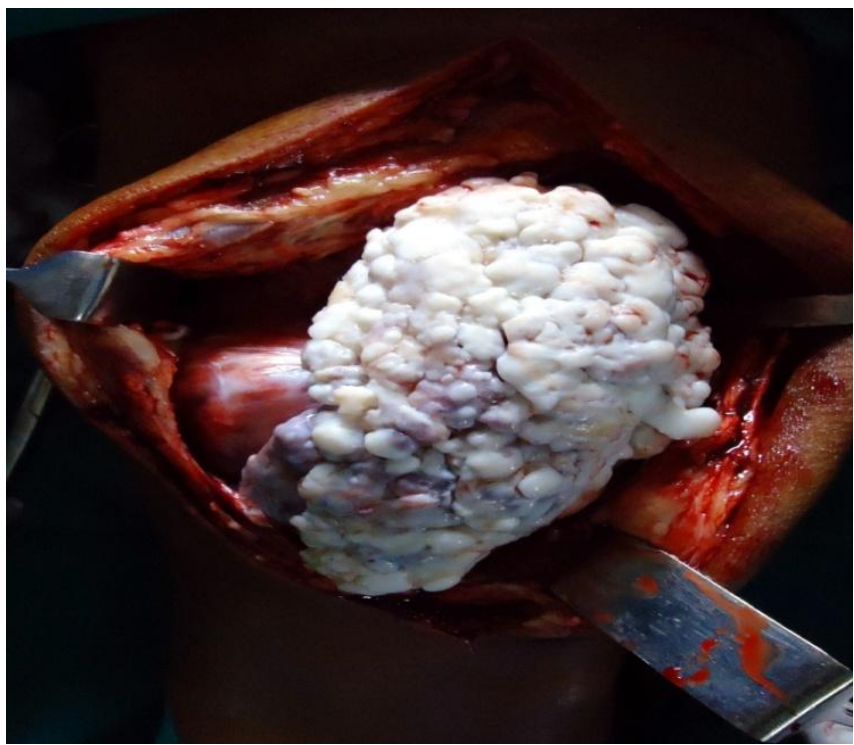

Figure 5 a Tumor Tissue

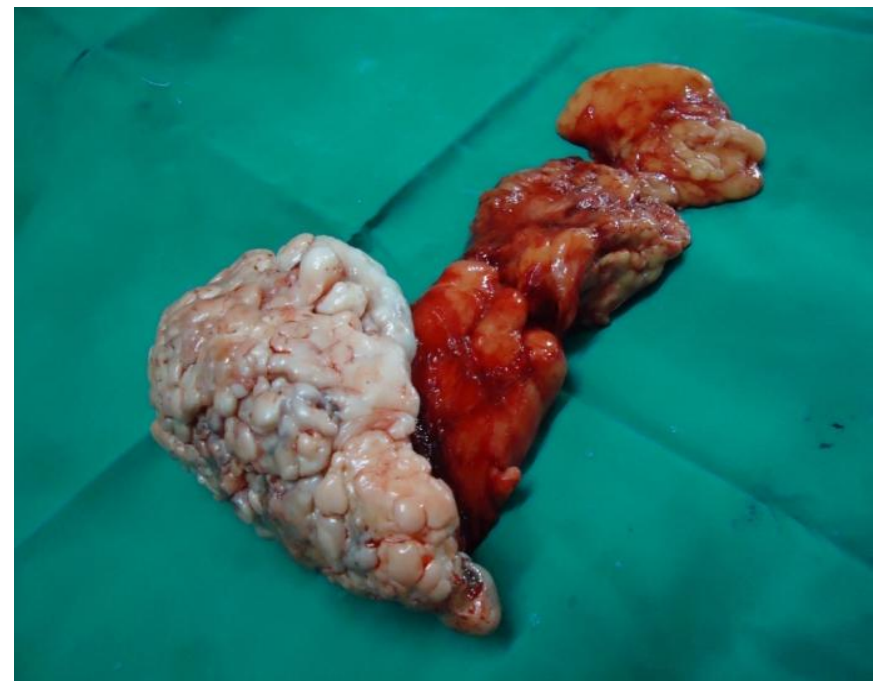

Figure $5 \mathrm{~b}$ Tumor Tissue

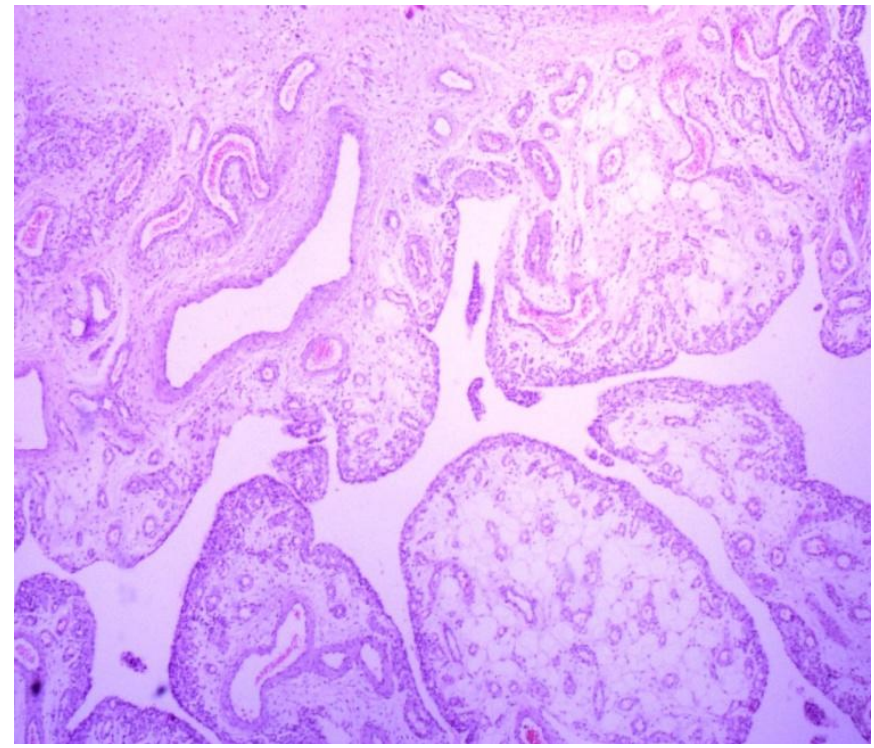

Figure 6 